\title{
Application of intelligent methods for diagnosing animal diseases based on determining the structures of blood facies
}

\author{
Marina $V$. Yanaeva ${ }^{1}$, Elena $V$. Kuzminova ${ }^{2}$, Nikita A. Akindinov ${ }^{1}$, Denis $V$. Osepchuk ${ }^{2}$, and \\ Marina P. Semenenko ${ }^{2}$ \\ ${ }^{1}$ Kuban State Technological University, Krasnodar, 350072, Russian Federation \\ ${ }^{2}$ Krasnodar Research Center for Animal Husbandry and Veterinary Medicine, Krasnodar, 350055, \\ Russian Federation
}

\begin{abstract}
The intensive development of computer technologies, the growing importance of the problems of intellectualization and decisionmaking determine the need for software tools that make it possible to increase the efficiency of laboratory diagnostics in veterinary medicine. An analysis of the structure of the solid phase of biological fluids (facies) is proposed as a universal method for assessing the state of homeostasis of an organism. The paper highlights the problem of diagnosing animal diseases based on the determination of indicators of the structures of blood facies, substantiates the relevance of using neural networks to recognize the obtained images of blood facies, presents the main methods of an intelligent information system for diagnosing animal diseases.
\end{abstract}

\section{Introduction}

The intensive development of computer technologies, the growing importance of the problems of intellectualization and decision-making determine the need for software tools that make it possible to increase the efficiency of laboratory diagnostics in veterinary medicine.

It has been scientifically proven that pathological processes already at the early stages of their development are accompanied by changes in the physicochemical properties of the internal environment of the body. An analysis of the structure of the solid phase of biological fluids (facies) is proposed as a universal method for assessing the state of homeostasis of an organism. This method is based on the theory of self-organization, according to which, during dehydration, the elements dissolved in biofluids form stable functional bonds that form specific structures characteristic of certain pathological processes. Crystallographic methods of laboratory diagnostics are economical, available for wide practice, capable of providing integral information about the state of the body with a high degree of reliability at the earliest stages of the development of pathological processes, which opens up broad prospects for this direction in clinical medicine and other areas of study of living systems [1,2].

However, the application of crystallographic methods in veterinary medicine (obtaining and describing crystallograms in pathological conditions in animals) is at an early stage. A limiting factor in the development of this promising direction is a certain difficulty in 
interpreting the crystal pattern in normal and pathological conditions, due to a significant variation in the types of crystal growth in a dehydrated drop of biological fluid.

The aim of this work is to substantiate the possibility of using neural networks in veterinary medicine for recognizing images of crystallograms of animal biofluids [3].

\section{Materials and Methods}

At the Krasnodar Research Centre for Animal Husbandry and Veterinary Medicine, a crystallographic study of the blood of healthy calves and those with colibacillosis was carried out. Whole blood sample was taken from animals, which, then, in the form of a drop was applied to a previously defatted glass slide and dried at $\mathrm{t}=20-25^{\circ} \mathrm{C}$, relative humidity -65 $70 \%$ and minimum mobility of the surrounding air, strictly in a horizontal position. The duration of the drying period (before the analysis of the structure) ranged from 18 to 24 hours. The study of the dehydrated drop - "facies" was carried out using an optical microscope "Mikmed-3" with a built-in digital camera "Canon" (5.0 Mpx). The resulting image was transmitted to the monitor screen.

\section{Results and Discussion}

A visual comparison of the crystalloscopy pictures showed (Fig. 1) that the features of the facies of sick animals relative to healthy ones include the following: the peripheral zone is characterized by a strip of dried plasma of a darker color; in the center there is a reduced destruction of the crystal pattern; reduction in the number of fractures; the number of cases of asymmetric, chaotic arrangement of fractures dominates over symmetric ones.

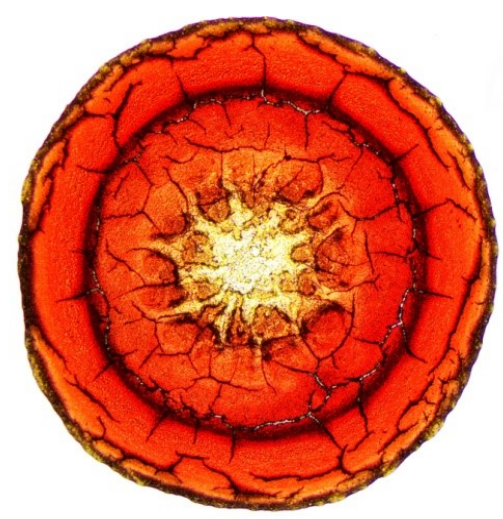

A -healthy

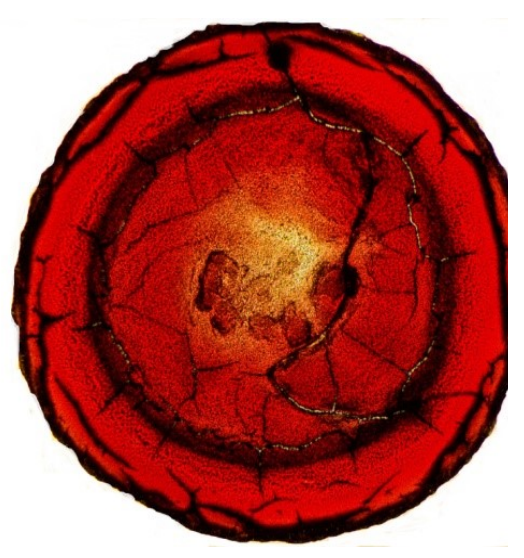

B -sick

Fig. 1. Crystalloscopic facies of calf blood (healthy and sick with colibacillosis).

When a biological fluid is dehydrated, the structure formation of the solid phase proceeds taking into account these relationships, which make it possible to analyze them.

Proceeding from this, conducting research to establish the relationship between the state of the body and the morphotexture of crystals of the corresponding biofluids, the formation of databases and knowledge bases on their basis, the construction and training of a convolutional neural computer network, makes it possible to develop a new information technology for recognizing the pathology of the body [4]. 
Currently, neural network technologies are used in humane medicine. Some of the most requested are listed below:

"Ideal anesthesiologist based on artificial intelligence" (2020) - a neural network developed by a group of scientists from the Massachusetts Institute of Technology and Massachusetts Hospital, which is able to monitor the patient's level of unconsciousness during surgery and determine / administer the optimal dose of anesthetic based on pharmacokinetic and pharmacodynamic models with random parameters. Testing has shown that the new algorithm outperforms the proportional-integral-derivative regulator, which is often used to determine the ideal dose of anesthetic. The main advantages of the new approach are the ability to scale clinical variables, as well as the relationship of the deep network with the input variables and the recommended dosage [5].

"Artificial Intelligence for Tuberculosis Diagnosis" (2019) is an AI system BacillAi, developed by the English company of Cambridge Consultants, which allows to partially solve the problem of the spread of tuberculosis in developing countries, where this disease is one of the most frequent causes of premature death. To diagnose tuberculosis using BacillAi artificial intelligence, a doctor only needs a microscope and a smartphone with Internet access. The doctor simply takes a photo of the patient's stained sputum sample with the application using a conventional laboratory microscope. Further, the image is automatically processed by a neural network on a remote server, and soon the doctor receives information about the number and type of tuberculosis cells in the sample.

"Botkin. AI - Russian AI system for diagnosing cancer" (2019) - a startup that develops an artificial intelligence system for automatic analysis of medical images and diagnostics of diseases. Botkin. AI already knows how to analyze CT scans of the chest. It provides the specialist not only with information about the detection of a tumor, but also highlights the areas that require its attention. The accuracy of the algorithm when analyzing a computed tomogram of the chest is up to $95 \%$. The system is improved not only by processing a large amount of images, but also in the process of practical work with doctors (when the doctor confirms or refutes the diagnosis). In the near future, the developers promise to add modules for the analysis of mammography and digital x-rays, which will increase the number of pathologies recognized.

In 2018, Google created AI to diagnose eye diseases. In medical centres, optical coherence tomography is used to diagnose eye diseases - a modern non-invasive non-contact method that allows visualizing various structures of the eye with a higher resolution (from 1 to 15 microns) than ultrasound. However, this technology has a significant drawback - it takes a very long time to process and interpret the images (in some cases, about a week), and in some conditions it can be fatal for the patient. The Google DeepMind team has created a neural network that can process these images very quickly and automatically detect the presence of fifty different eye diseases. The neural network was trained on a huge database of images from the British Eye Center Moorfields Eye Hospital, verified by qualified specialists.

In 2018, Google also created an augmented reality microscope for diagnosing cancer, as examining blood samples under a microscope is still a necessary procedure for diagnosing cancer. Moreover, the accuracy of the diagnosis depends on the doctor's eyes. For this procedure, Google employees developed a neural network trained on millions of already processed samples. Their augmented reality microscope system automatically draws the doctor's attention to suspicious areas, so the diagnostic accuracy is significantly increased. The development allows real-time analysis of the image and presentation of the results of recognition of the cells with which the study is carried out. The image refresh rate is 10 frames per second, which is quite enough for diagnostics. The algorithm itself is based on machine vision and machine learning technologies. 
An intelligent information system for the diagnosis of animal diseases will be implemented on the basis of a convolutional neural network. The following are the planned ways to implement an intelligent information system for the diagnosis of animal diseases $[6,7]$.

Wavelet large-scale analysis, texture analysis of images, segmentation method, spatial methods of image enhancement, digital image processing algorithms, one-dimensional signal processing, statistical data processing, system analysis, and pattern recognition are used as digital image processing methods. Wavelets are widely used for filtering and preprocessing data and analyzing state, pattern recognition, for solving problems of image compression and processing, for training neural networks. Our vision is designed in such a way that we focus our attention on the essential details of the image, cutting out the unnecessary. Using the wavelet transform, you can smooth or highlight some of the details of an image, enlarge or reduce it, highlight important details, and even improve its quality.

Segmentation methods can be divided into two classes: interactive - using user input directly in the process of work and automatic - not requiring any interaction with the user. For morphological processing of images, methods of biomedical morphometry, morphological filtering, thinning and truncation will be applied.

A neural network is a mathematical model, as well as its software or hardware implementation, built on the principle of the organization and functioning of biological neural networks - networks of nerve cells of a living organism. An artificial neural network is a system of interconnected and interacting processors (artificial neurons). Such processors are usually quite simple (especially when compared to processors used in personal computers). Each processor on such a network deals only with signals that it periodically receives and signals that it periodically sends to other processors. And, nevertheless, being connected in a sufficiently large network with controlled interaction, such individually simple processors together are capable of performing rather complex tasks $[4,6]$.

From the point of view of machine learning, a neural network is a special case of pattern recognition, discriminant analysis and clustering methods. In terms of mathematics, training neural networks is a multi-parameter nonlinear optimization problem. Neural networks are not programmed in the usual sense of the word, they are trained. Learning is one of the main advantages of neural networks over traditional algorithms. Technically, training consists in finding the coefficients of connections between neurons. During training, the neural network is able to identify complex dependencies between input and output data, as well as perform generalization. This means that in case of successful training, the network will be able to return the correct result based on data that were absent in the training set, as well as incomplete and / or "noisy", partially distorted data.

The main element in image processing using neural networks is analysis, the first phase of which will be image recognition. After that, artificial intelligence using machine learning recognizes the actions and classifies them.

In order to recognize an image, the neural network must first be trained on the data. This is very similar to the neural connections in the human brain - we have certain knowledge, see the object, analyze it and identify it $[8,9]$.

Neural networks are demanding on the size and quality of the dataset on which it will be trained. The dataset can be downloaded from open sources or collected on your own.

A neural network for image recognition is perhaps the most popular way to use neural networks. At the same time, regardless of the features of the tasks being solved, it works in stages (Fig. 2). 


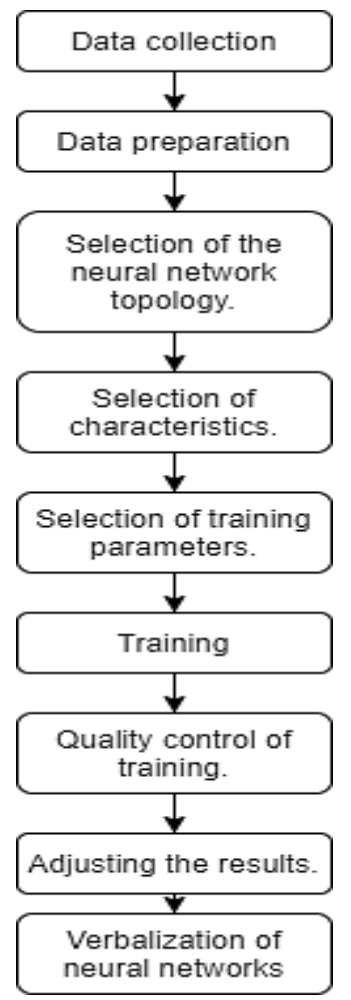

Fig. 2. Scheme of creating a neural network for image recognition.

The images of the crystallographic facies of the blood of animals will be the recognized images. When training a network, various samples with a label of what type they can be attributed to are offered. A vector of feature values is used as a sample, and the set of features under these conditions should allow to unambiguously determine which class of images neural networks deal with $[10,11]$.

When training, it is important to teach the network to determine not only a sufficient number and values of features to give good accuracy in new images, but also not to overfit, that is, not to "adjust" unnecessarily to the training set from images. After completing the correct training, the neural network should be able to identify images that it did not deal with during the training process.

It is important to take into account that the initial data for the neural network must be unambiguous and consistent, so that situations do not arise when the neural network will produce high probabilities of belonging of one object to several classes.

A convolutional neural network $(\mathrm{CNN})$ contains one or more concatenated or connected convolutional layers. CNN uses a variation of the multilayer perceptron. Convolutional layers use convolution on the input and pass the result to the next layer. This operation allows the network to go deeper with fewer parameters. Convolutional networks show outstanding results in applications requiring image recognition. It is the convolutional neural network that will be built and trained in the intelligent information system for the diagnosis of animal diseases [12].

\section{Conclusion}

The main expected scientific results are: 
- development of methods for diagnosing diseases based on the determination of indicators of the structures of blood facies in animals;

- building and training a convolutional neural network;

- development of methods for recognition of medical images (facies of biofluids);

- building an intelligent information system for the diagnosis of animal diseases.

\section{References}

1. A.I. Andyushkin, Bulletin of the Chuvash University, 3, 355-9 (2013)

2. V.N. Kidalov, A.A. Khadartsev, Tula polygraphist, 244 (2009)

3. S.A. Kraevoy, N.A. Koltovoy, Electronic Mathematical and Biomedical J.

"Mathematical morphology", 320 (2016)

4. G. Aurelien, OOO Dalektika, 688 (2019)

5. S. Kraevoy, N. Koltovoy, Electronic Mathematical and Biomedical J. "Mathematical Morphology", 1, 321 (2016)

6. V.O. Petrov, V.A. Kamaev, S.V. Poroisky, Modern problems of science and education, 6, 106-10 (2009)

7. G. Sreenu, M. A. Saleem Durai, J. of Big Data, 6 (1) (2019)

8. S. Francois, Peter, 400 (2018)

9. A. Trask, Peter, 352 (2020)

10. A.S. Bolkhovitinov, A.G. Verhovcev, O.V. Gradov, Electronic Mathematical and Biomedical J. "Mathematical morphology", 9 (1) (2010)

11. R-C. Mihailescu, P. Davidsson, U. Eklund, J.A. Persson, Knowl. Eng. Rev., 33, (2018)

12. C. Szegedy, et al., MA, 1-9 (2015) 